\title{
Monitoring \& Controlling of Information against Unethical Hacking using Effective Machine Learning Techniques
}

\author{
Sudheer Pullagura, S.V. Naga Srinivasu
}

\begin{abstract}
Many services are currently utilizing AI estimates to pick high-stake options. Determining the proper selection unequivocally relies on the rightness of the relevant information. This fact offers encouraging motivators to hackers to attempt to mislead Artificial Intelligence estimations through managing the relevant information that is taken care of to the estimates. But at that point, standard AI computations are certainly not wanted to become protected while encountering surprising details resources. At the moment, deal with the concern of ill-disposed AI; i.e., our experts will most likely generate risk-free $A I$ calculations robust within the attraction of a loud or an adversely managed information. Ill-disposed Artificial Intelligence will be even more screening when the perfect turnout has a mind-boggling framework. At this moment, noteworthy limelight gets on adversarial AI for preparing for organized returns. To begin with, our team build up yet another calculation that dependably carries out accumulated collection, which is an organized expectation concern. Our discovering approach works and also is described as a curved square system. This method is sure about the desire calculation in both the closeness as well as the absence of an opponent. Next off, our team looks into the problem of criterion learning for strenuous, coordinated projection models. This technique develops regularization capacities dependent on the restrictions of the adversary. Now, illustrate that durability to the command of details corresponds to some regularization for a tremendous edge arranged assumption and the other way around.A typical device commonly either requires more computational capability to structure a clearcut best assault, or it doesn't have adequate records about the trainee's design to accomplish, therefore. Consequently, it routinely tries to use many unnatural changes to the payment to a desire to bring in an accomplishment. This reality advises that on the occasion that our experts confine the usual lousy luck job under illdisposed commotion, we will get vitality against ordinary opponents. Failure preparing seems like such an outcry mixture circumstance. Our experts calculate a regularization technique for an enormous edge parameter, discovering depending on the failure system. We stretch out dropout regularization to non-straight parts in a handful of one-of-a-kind means. Empirical analyses show that our systems reliably pounded the standards on a variety of datasets. This proposition integrates a recently dispersed and individual coauthored component.
\end{abstract}

Keywords: Approximation quality, Reinforce vector machines (SVMs), Kernel approximation, Rundown of commitments

\section{INTRODUCTION}

Central trouble in Artificial Intelligence is understanding intricate types that summarize to undetected reports.

Revised Manuscript Received on June 15, 2020.

Corresponding Authors*

Sudheer Pullagura*, Research Scholar, Computer Science And Engineering , Acharya Nagarjuna University, Nagarjuna Nagar, Guntur, Andhra Pradesh, India.

S.V. Naga Srinivasu, Professor, Dept. Of CSE , Narasaraopeta Engineering College, Narasaraopet, Andhra Pradesh, India.

(C) The Authors. Published by Blue Eyes Intelligence Engineering and Sciences Publication (BEIESP). This is an open access article under the CC BY-NC-ND license (http://creativecommons.org/licenses/by-nc$\underline{\mathrm{nd} / 4.0 /)}$
One outlook of the task is to utilize the clothes of countless versions in contrast to a single layout. One more system is actually to find yourself being the dataset, either clearly and even plainly, through battering invariance's in the room. The two approaches reduce the difference between the estimator, frequently irritating sound styles. Dropout prepping could be thought about as a case of each of these methods. In desertion readying, pieces of the model or info are subjectively left" while determining the requirements. Along these lines of product, a breakdown can be considered increasing the transportation of models, and even upgrading a model on a dispersing over datasets. In noteworthy frameworks, this lowers co-change of the loads and allows dynamically intricate styles to end up being scholastic and a lot less over-right. In surface styles, for example, important backslide (LR), failing handles as a regularizes that pushes back incorporate problems reliant on the total amount they affect the classifier's assumptions within this term paper. [1] Reinforce vector machines (SVMs) are among the most distinctive and successful distinction approaches; obtaining the highest fee realizes many places. SVM preparing yourself matters lower principle bungle by boosting the (at risk) conveniences between the courses. For straight classifiers, this is of consequence limiting the turning issue regardless of a square bodyweight regularizes. To acquire capacity along with a non-straight classifier, SVMs may quickly make use of a region potential to figure contact think about a high-dimensional part area without building up the accurate part picture. Simultaneously, the best side suggestion helps enhance the concept; overactive continues to be a risk when appreciating intricate limitations stemming from the required information. Kernelized SVMs are at the complete most actual danger, as a result of their expanded expressivity. Previous solution breakdown has often based upon significant systems and also identified backslide. For calculated backslide, there are units to create preparing yourself much more reliable through predicting or even thinking little of over the incongruity provided via breakdown. Different files sever the quantitative as well as the mental impact of failing in learned backslide. The major handle breakdown in SVMs is constrained to direct SVMs as well as entails a tolerably snared method for moving the low breakdown target. [2] At today's opportunity, take into consideration failure in both straight as well as likewise nonstraight SVMs. Our team is going to produce methodologies that are direct, reputable, possibly, and also reliable at strengthening the conjecture of SVMs on actual industry datasets.

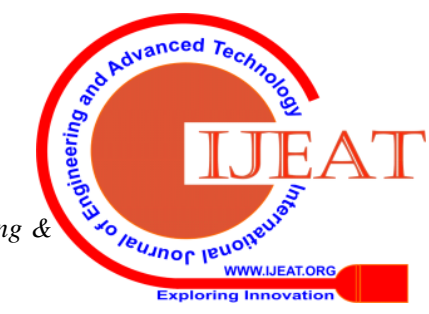


For straight SVMs, our provider presents that the typical convert case under failure difficulty could be immovably approximated as a smooth, shut structure task. This minimal failure purpose is most definitely undoubtedly easy to ravel and also hints improved completion on various datasets.

For non-straight SVMs, our crew program two techniques for successfully doing failure on the part feature chart, regardless of when this market map is high or infinitedimensional. Our first function creates a quick image of the info through aimlessly assessing from the Fourier renovation bases of the component function as delivered within this research paper. It by then recognizes a straight SVM alongside restricted failure mayhem on this altered component photo. The observing strategy estimates the result of dropout in the private area by incorporating a heavy L2 regularizer to the two-fold factors in the SVM progress concern. In tests finger distinction and also analysis datasets, both devices lead to far better execution seemed to be in various methods regard to a normal SVM together with a sturdy blowing winding description job (RBF) element, anyway the customized component image approach is a whole lot a lot more trustworthy than pair of fold regularization. [3]

\section{RELATED WORK}

The connection between various kinds of disruption and additionally, regularization has been checked out at with multiple creators. As an example, Diocesan (1995) presents that integrating Gaussian smashing to nerve organs structure inputs while preparing yourself is, in fact, proportionate to L2 regularization of the heaps. For the scenario of direct SVMs, current that most cynical circumstance features component clatter in addition to constrained standard amounts regularizing the bunches in addition to the dual norm. Found the issue at examination opportunity" scenario in which an opponent kicks out a particular selection of functionalities originating from the concept, establishing all of them to no [5] They suggest a transformed SVM indicating to reinforce completion against such an adversary. It split the regularization influence of failure noise in summarized direct styles (GLMs) through securing a second-demand idea to the usual loss of the dropoutsabotaged maximums. This makes it possible for the breakdown emphasis on coming to be improved positively as opposed to entirely. Incredibly, this second-demand scale can easily not be put on coordinate SVMs, taking into account how the to revolve collision isn't differentiable.

Maarten and the like additionally existing approaches for figuring out the right styles and polluted attributes, confining over the destruction via launching a surrogate upper bound of the well-established mishap. For certain accident limits and uproar apportionments, they may conveniently participate in the put-down goal positively; for worked out events, they restrain a higher linked on the common challenge. [4] They don't think about turn issue. Relax these methods to review straight SVMs in addition to failure noise. Because of the reality that is appropriately tapping the services of the concept, little of intended is challenge some; the developers offer a variational surmise. They smooth out this estimated focus on using desire growth in addition to iterative the extremely minimum squares. The spots of Chen and so on resemble our personal. However, our agreement is less challenging and a lot less harsh to ravel. Wang and additionally Manning obtain knowledgeable a strenuous method along with secured the usual breakdown slant. The necessary principle is to pull the noised company of each device coming from a frequent scattering instead of drive, taking into consideration various Bernoulli aspects. By utilizing this scale several times for every readiness version, the reputation in the conveniences is decreased without a sizable boost in the matter option. They, in like manner, present a sealed construct tactical plan, which counts on approximating the important stipulation as a Gaussian complete scattering job. At the here and now, an opportunity, in like manner, utilize a Gaussian stab in the dark to the uproarious bit variables. All the same, our staff base upon turn obstacle as opposed to determining hardship, and our team urge the superb ideal strategy to figure procedure the desire smartly without thinking about or perhaps introducing any other estimates. Failing is, in fact, considerably different from highlighted material uproar as a result of the simple fact that the typical nuisance of a portion relies upon its inspiring force in the data. As an example, features that are presently ultimately no are going to be annoyed via requirement featured drug upheaval most definitely, nonetheless, remain unaltered by failure. Or perhaps possibly, dropout interruption is most beautiful seen as an occasion of multiplicative noise due to the reality that every aspect is increased through 0 along with some option as well as one =-LRB- 1) together with a likelihood (1). [6] Till this point in time, there has been encouraged analysis of prepping alongside multiplicative racket besides dropout1, and also no evaluation of arranging SVMs along with multiplicative interruption. Currently, take care of each of these requests, inciting rampant attention of only how outcry relates to the hypothesis in a different type of layouts.

\section{APPROXIMATION QUALITY}

Since the approximation depends on the central limit theorem, (assuming that $\mathrm{z}_{\mathrm{i}}=1 \mathrm{y}_{\mathrm{i}}\left(\mathrm{w}^{\mathrm{T}} \mathrm{x}_{\mathrm{i}}+\mathrm{b}\right) \mathrm{N}\left(\mathrm{u}_{\mathrm{i}} ;{ }_{\mathrm{i}}{ }^{2}\right)$ ), this method should be used when the data is not extremely sparse (e.g., there are at least 10 non-zero features in the average sample), and the regularization penalty does not favor extremely sparse solutions.

More formally, let $\mathrm{u}_{\mathrm{i}}=1 \mathrm{y}_{\mathrm{i}}\left(\mathrm{w}^{\mathrm{T}} \mathrm{x} \sim_{\mathrm{i}}+\mathrm{b}\right)$ be a random variable that represents the margin for some fixed weights $\mathrm{w}$ and some arbitrary dropped-out sample $\mathrm{x} \sim_{\mathrm{i}}$ with the desired label $\mathrm{y}_{\mathrm{i}}$, and $\mathrm{m}_{\mathrm{w}}$ be the number of non-zero elements in $\mathrm{w}$. Also let $\mathrm{F}_{\mathrm{ui}}(\mathrm{z})=\mathrm{P}_{\mathrm{ui}}\left(\mathrm{u}_{\mathrm{i}} \mathrm{z}\right)$ be the cumulative density function (CDF) of $\mathrm{u}_{\mathrm{i}}$. By the Berry-Essential theorem, the supremum of the difference between the CDF of $\mathrm{u}_{\mathrm{i}}$ and its Gaussian approximation is upper-bounded by:

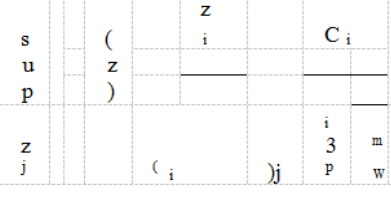

As a result of the complete best quote to time, $\mathrm{C} \mathrm{0:4748.} \mathrm{i}$ is the 3rd instant of UI, as well as might be computed in closed-form.

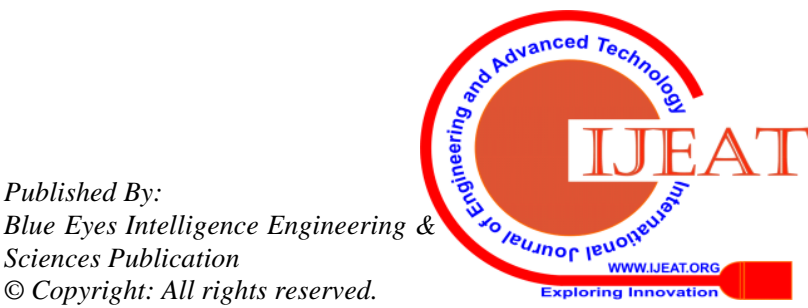


In Figure 5.1 b, our staff replicates this upper-bound for a variety of numbers of non-zero weights on a plaything dataset. In practice, we monitor that truth as well as likewise the approximated distributions of UI carefully match each other as within this term paper.

It is quite simple to verify that the optimization strategy in (Formula 4) is always a leading player connected to the conventional SVM's goal. Because of this, the failing estimation dwells in easy truth, advertising, and marketing transmission that inherently utilizes added regularization results on the figured out physical body weights. The objective is a soft approximation of a convex component (the prepared for hinge-loss). It is also easily varied in addition to taking full advantage of pitch inclination, LBFGS, or even numerous other conventional methods. [8] Our team offers visual preference regarding our created estimate. Our pros take a look at one single sample, and also reveal the joint decrease (reddish), its sealed type requirement arising from Equation 3 (eco-friendly), and also the Monte-Carlo feature when the functionality is averaged over real failure loud examples (blue). The noised joint reduction supplies a top tied that is tight at the extremities as well as also improve in between. It shows just how several examples with different ranges develop the aggregated reduction functionality. As the dimensionality of design body system weights raises, the estimate firmly combines to truth requirement, which is convex. For incredibly lowdimensional inputs (4-5), the strategy may still be applied but can perform poorly. This procedure is appropriate for real-world complications, where our experts take care of hundreds and even 1000s of dimensions.

\section{Kernel approximation}

Although kernel methods have revealed to end up being successful in non-linear predictive styles, recognizing these models asks for $\mathrm{O}\left(\mathrm{n}^{2}\right)$ mind along with a prolonged direction time, and also calculating the collection functionality may be pricey when the amount of support angles is sizable. These two issues create part strategies much less practical, specifically on substantial datasets. Randomized methods for estimating piece matrices (Scholkopf, 2002; Blum, 2006) have determined countless ways to change the guideline and study of part devices appropriately properly into straight bodyweight discovering and credit score ranking prophecy. The keynote responsible for these approaches is actually to situate a reasonably low-dimensional quality representation $z(x)$ such that $z(x i)^{T} z(x j)$ estimates the preferred bit functionality, $\mathrm{k}(\mathrm{xi} ; \mathrm{xj})$.

Besides the functional performance of such feature portrayal procedures, our professionals can conveniently make the most of a lot more intricate straight techniques to enrich the projection. As an example, this is going to allow our provider to typically use the marginalized upright SVM approach within this research paper.

Our team has boosted Rahimi and also Recht's technique by focusing on the RBF bit. Possessing said that, it could be related to every other translation-invariant piece as well. Their method is based upon Buchner's theorem: A recurring translation-invariant piece $\mathrm{k}(\mathrm{xi} ; \mathrm{xj})=\mathrm{k}(\mathrm{xi} \mathrm{xj})$ on $\mathrm{R}^{\mathrm{d}}$ declares guaranteed if and merely if $k()$ is the Fourier fully transform of a non-negative solution." By randomly testing arising from the regards to this Fourier remodeling, we may find easily comparative the bit in addition to some confluence guarantees.
Monte-Carlo dropout in input space and dimension

Our experts may conveniently generate a Monte-Carlo evaluation of Equation 5.11 through changing out the requirements overall failure audio alongside $\mathrm{K}$ examples of failing noise for each directive celebration. This amounts to getting from countless loud copies of the instruction info. For input location dropout, our team can quickly generate several rowdy copyings of the training information as well as give standard SVM learning algorithms. This runs considering that input space failure uses audio just before calculating the little [9]. For input measurement failure, our company needs to make a note of the dropout noise clearly and also utilize it to personalize the bit evaluation. As an example, for the RBF kernal., $k\left(x_{i} ; x_{j}\right)=e^{k x_{i} x_{j}{ }^{k 2}}$. When applying dimension dropout, we need to modify the distance computation so that it only considers non-dropped-out

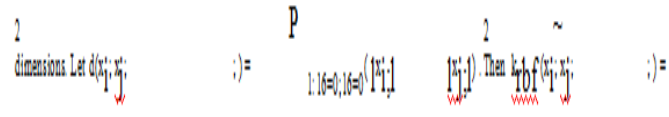

\section{e digl'}

To apply this successfully, our group exemplifies xi and likewise $\mathrm{xj}$ as thin slants where all unspecified sizes are really stopped, plus all non-dropped-out nos are written explicitly. In the piece computation, our group is loyal just over measurements where each xi and xj have an established market value (which might ultimately be no).

The vital perk of the input dimension failing is that it maintains the translation-invariance residence of $\mathrm{RBF}$ pieces. The essential negative aspect is actually that the leading little bit source might be non-PSD. In our practices, our team located that input size dropout beats the regular RBF kernel. Also, the negative eigen market values of the particular piece were, in fact, usually pretty small in level, along with performed certainly not induce any user issues for the minimal sequential advertising (SMO) algorithm. If essential, tactics for maintaining the optimization of nonPSD kernels might be used below also (Lin, in addition to Lin, 2003). For RBF items, a model academic alongside failure might work poorly on non- significant events. Our crew administers a pair of numerous techniques in our exams. [10] The initial is really to forget this difference as well as likewise carry out the style straightforwardly. For a few failure odds $(5 \%-10 \%)$, the new vulnerability should be a tiny bit of. The subsequential strategy is actually to figure the regular section to persuade all possible dropout fury. Because every failure odds is free of charge, this has to be doable in straight opportunity. (The verification is given up Appendix E.) Our experts discuss this latter method as the boosted" projection. In each condition, dropout chaos is offered through expelling arbitrary highlights along with surely not rescaling the remainder of the highlights; the rescaling melioration ( $1=$-LRB- 1$)$ ) is planned for direct models along with generates troubles when improving angles and also test cases are scaled differently.

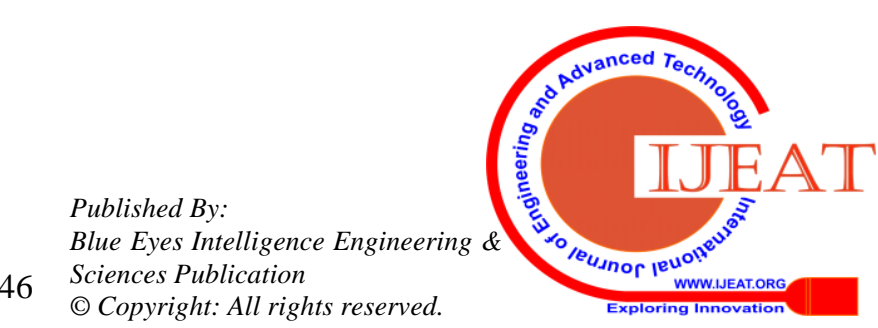




\section{Exact Outcomes}

Datasets. We ran our exams on a couple of hand-operated type datasets, the MNIST finger type dataset, and the Adult dataset from the UCI storehouse. The material datasets were pair of believed examination datasets revealed using Discomfort as well as likewise Lee, three datasets depending on 20-newsgroups lately used with Wang and additionally Manning, as well as additionally 4 Amazon.com slant datasets. Our experts moreover created an artificial dataset that contacted M27 coming from MNIST. In M27, our experts have decided on every one of the two and also seven figures from MNIST. For every single digit, our experts haphazardly chose two-digit amounts, at that point indicated all pixels that match up to the files arising from $\mathrm{I}$ to $\mathrm{j}$ of the vectorized 784-dimensional figure image to no. Our company rework this for the planning, the adjusting, as well as additionally the evaluating info. [10] Procedures. On the info datasets, our main problem is actually to show the around exhibition of different primary SVM-based techniques: our crew take into consideration the minimized (SVM-Marg), Monte-Carlo failing (SVM-MC), and also regularization (- Reg), during with essential parts (Table 1). For nonlinear elements, our experts concentrate on outspread residential or commercial property abilities (RBF). Our crew considers various direct techniques that use the relative Fourier etiquettes as attribute duplicate in addition to that ordinary SVM and also our prepared regularization procedure (Table 2).

Exploratory System. For the web material distinction examinations, our team made use of ve furrow cross commendation. For the Monte-Carlo strategies, our business generates $\mathrm{K}$ matches of the prepare work information as well as likewise utilize breakdown chaos to every instance quickly. In addition to uproarious duplications of the planning info, learning is an evaluation of limiting the common woe when the outcry parts are thoughtlessly drawn from their distinct publication. All hyper-parameters are chosen with cross-approval. For the approximated bit makes an effort in Table 2, our professionals set up the component of Fourier etiquettes D $=4000$ for MNIST along with M27 datasets, and $\mathrm{D}=1500$ for the Adult dataset. All the same, our pros tuned all a variety of other hyper-parameters, utilizing held-out relevant information. At that point, reprepared the final style by featuring both the planning and adjusting info evaluations. [12] Nonlinear forms (without straight estimate) are progressively vulnerable to hyperparameters. For that reason this fact, our staff have really furthermore tuned 2 for the nonlinear component regularization method, similar to the '2 regularization coefficient, and also the RBF component rule for all purposes. Then again, the readjusting unit commonly was chosen much bigger failure odds for upright (each straight as well as a stand-up hunch of RBF) variations.

Outcomes: Table 1 reveals the blunder degree of each straight classifier on every one of the content datasets. The best-performing variety is appeared in striking. [11] Marginalized failing beats all several other methods-except in one dataset, on which regularization outperforms SVMMarg. Monte-Carlo failure instruction induced enriched results on all datasets.-Reg Small makeovers on 7 of nine datasets, however, commonly ran considerably worse than SVM-Marg, suggesting that marginalization in the primitive is extra dependable when suitable. Our specialists have additionally distinguished our approaches together with logistic regression, LR with Monte-Carlo failure, and also LR with (marginalized) deterministic dropout.

The outcomes have a pure audio design, whenever SVM by itself exceeds LR, the SVM-based failure procedures likewise elude the LR-based failing techniques, and viceversa.

\section{RE-POSITIONING AND SEARCH-BASED STRATEGIES}

Re-positioning is mainly applied to the usual different language managing troubles. Anticipate that our team resembles the that cares for some reduction complication; however, rather than creating \the best" turnout, it generates a review of $n$ most exceptional" gains. At that point, he's trainee will certainly more than likely create a second model for deciding on one yield" from the $\backslash n$ finest" returns. A second concept then enriches this rooting positioning, making the most of incorporated highlights as evidence. This approach makes it possible for a tree to be consulted with as a discretionary program of highlights, without fret about precisely how these highlights users interface or cover, as well as also without the necessity to de ne an expectation which thinks of these highlights.

Re-positioning has been administered in an assortment of NLP problems featuring parsing, and additionally numerous tasks. A central aspect of re-positioning is that different misfortune capabilities might be smoothly put into the estimation and additionally rapidly made an effort. There are moreover a few negative aspects. As an instance, in a repositioning evaluation, one should possess a for selecting nbest marketing positioning, which might undoubtedly not come, and even n could also be substantial ever before to be beneficial. [Thirteen] Search-based organized insight could be taken into consideration as an improved and additionally additional industrialized variety of re-positioning. The re-authorization learning body generally elevates these calculations and likewise possess a favor of managing the dealt with forecast issues from a coordinating standpoint. Revealed search-based collaborated requirements along with the SEARN evaluation. This estimate mixes appearing and also identifying precisely how to handle work with belief troubles. LEARN is a meta-calculation that changes coordinated wish issues right into simple type issues, to which any sort of form of the same classifier may be used. SEARN might learn forecast capacities for various misfortune capabilities as well as additionally different highlights abilities. Several other linked jobs apply relative devices [6] Greatest edge Markov systems.

The ideal edge Markov arranges (M3N) course of organized forecast methods is an opinion of max-edge strategies in well-known AI (typically telephoned assistance vector tools (SVM)) to arranged yield presumption settings. The quite first job was transmitted using a sizable volume of new developments being produced of max-edge methods.

To date, the modern architectural SVM is the 1-slack service that remedies the observing advertising and marketing strategy:

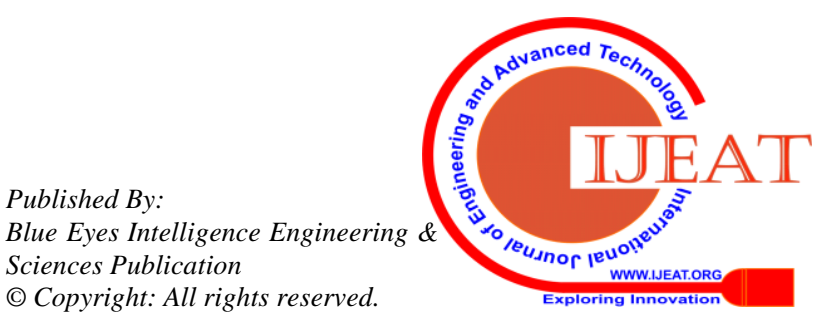




\begin{tabular}{|c|c|c|}
\hline \multicolumn{2}{|c|}{ minimize $\mathrm{f}(\mathrm{w})+\mathrm{C}$ subject to } & (Equation 8) \\
\hline $\mathrm{W}$ & & \\
\hline & $\left.\max \mathrm{w}^{\mathrm{T}} \mathrm{(}(\mathrm{x} ; \mathrm{y} \sim) \mathrm{f}(\mathrm{x} ; \mathrm{y})\right)+(\mathrm{y} ; \mathrm{y} \sim)$ & \\
\hline & $y^{\sim}$ & \\
\hline
\end{tabular}

$\mathrm{f}(\mathrm{w})$ is a regularization function, that penalizes large" weights. Depending on the application, $\mathrm{f}(\mathrm{w})$ can be any convex function in general. Semi-homogeneous functions, such as norms, or positive powers of norms are among the favorite choices ${ }^{2} . \mathrm{f}(\mathrm{w})=\frac{1}{2} \mathrm{w}^{\mathrm{T}} \mathrm{w}$ is the most commonly used regularization function. For simplicity, I have disclosed the input records as a solitary instruction instance. Still, it may conveniently be included point out of $\mathrm{N}$ specific examples, each of that creates a personal repayment to the reduction function. The variable is, in fact, the only slack variable, which ought to be lessened, alongside the regularization capability.

The large-margin Markov networks are cultivated as convex marketing programs. Subsequently, it is mathematically functional to secure sturdy solutions based upon every one of them. Within this line of reasoning, we mainly focus on large-margin methods.

\section{Optimization Algorithms}

In a number of the strategies that our professionals showed above, the learning algorithm is mounted into the design, having said that, for the max-margin techniques, our team generally thinks of a mathematical optimization program. In the following, our crew brie y expose 2 of the modern optimization algorithms that are utilized for structured learning.

\section{Cutting plane calculation:}

2A work $\mathrm{f}(\mathrm{z})$ is actually semi-homogeneous if and just if $\mathrm{f}($ $\mathrm{az})=\mathrm{a} \mathrm{f}(\mathrm{z})$ for some advantageous. In criterion learning of the optimum side coordinated strategies, the objective is to decide on the rules for which the score of the legitimate verifications is put together greater than the ball game of every alternative name. Hypothetically, this should be possible using a raised growth course, for example, a square system. The trouble is in fact that the quantity of swap titles is typically remarkable in the relevant information measurements; because of this, submitting every one of each of all of them is unchecked. The cutting plane calculation at every pattern locates the other advertising that is most various arising from the genuine identifying in addition to possesses the complete most elevated credit history ranking, then includes the right restrictions to ensure ballgame of the legit identifying is reasonably more than this replacement naming.

\section{Column age:}

Our experts can easily recognize the bent system produced by the ideal side strategy in its dual style. The twin remodeling program possesses a relative difficulty where the volume of the double aspects is, in fact, quick in the applicable info measurements. Like the cutting plane calculation, the sector age strategy chooses a dual variable at every importance and also incorporates it into the dual course. Taking care of the trouble in its double structure is handy, taking into consideration the straightforward fact that afterward, our experts can make use of the magnitude of product capacities. There are, in fact, a couple of jobs that utilize section grow older for spec learning.

\section{Exponentiated slope}

The exponentiated slope computation also knows the redesign plan in its double construct and uses a perspective climb estimate for every upgrade in every usefulness. The critical point in the estimation is really that the angle of inclination is exponentiated (as an instance, it took advantage of in contrast to the inclination g), as well as also there are installment speculations every bit as exploratory analyses that display the productivity of this particular approach.

\section{Unfavorable AI}

Today, referring to the theoretical body of hostile AI when all is discussed in performed, and additionally concurrently deal with the simple part of the here and now job that placed on handled forecast complications. AI checks out AI approaches that are difficult against ill-disposed components, which rule over the operation of info particulars age. [8] As surveillance troubles are broadening, the demand for hostile AI price quotes is becoming gradually evident nowadays. In resemblance with safety and security and also protection concerns, ill-disposed AI could be thought about as a game in between two players, where one gamer requires to need to promise the routine benefit of design, and also the other player needs to must demand its evil-minded purposes. In AI phrasing, the initial gamer is called the pupil (or maybe the guard), and also, the subsequential player is known as the foe (or the aggressor).

There has been a much getting to a selection of work since past due that analyzes the security and protection of $\mathrm{AI}$ frameworks; this assortment features various training courses of potential assaults versus AI. In the going along with subsection, our expert's brie $y$ manage the comprehensive, very most considerable portion of the most useful lesson methods, along with our provider, will evaluate the critical subjects in ill-disposed AI quotes. Our team will most definitely also examine lament decline estimations, which are reasonably relating the ill-disposed AI. In the lament minimization body, Attribute simulates an adversary as well as collections the expenses and also rewards. The goal is actually to choose a pattern of activities that restrains the potential lament. Lament is figured out as the whole of all obtained expenditures of picked duties at unparalleled developments, tiny the overall of the prices when only one best-fixed job or even setup had been taken whatsoever the parties. The best-fixed task will be the one that would have been decided on if the entirety of the expenses were recognized remembering. [9] Now, standpoint usually is stemming from the student's point of view, along with our pros, prepare the hostile strikes based on additional significant level houses of an adversary. For a substantial variety of would-be risks that make a large section of the out-of-date layout AI estimations powerless against hostile attacks mention Nelson (2010 ). Our experts begin this portion with some interpretations: Opposing enemy in addition to lose-lose problems: The adversary's functions are solely against the apprentice; for example, the foe's excellence in a similar way shows the apprentice's misfortune along with the other way around. 
These video games are called lose-lose, as well as such an opponent is pertained to as an unpleasant enemy.

Non-opposing rival as well as non-lose-lose health conditions: If the competition's goals are valid versus the trainee's goals, at that point, the adversary is searching for its benefits, which could likely be effectively detrimental to the apprentice. At whatever objective, the activity of equal awards and also catastrophes of each aspect of the video game are, in fact, undoubtedly not undoubtedly similar; at that point, the video game is non-lose-lose. When it concerns boosting the student's expenditure, it isn't the vital idea of the opponent; then it is a non-hostile opponent.

Emphasizing the non-lose-lose situation is generally straightforward. Leave $\mathrm{w} 2 \mathrm{~W}$ alone the rules of the apprentice's style, as well as a 2 A, be the criteria of the opponent's style, where the enemy correctly and influences the show of the AI calculation. W and likewise An is actually separately the job room for the pupil and additionally the enemy. On top of that, permit ra( w; a) be the disaster job that the pupil demands to restrict with picking the possibility w3. A standing up to opponent needs to have to magnify the decrease of the pupil through deciding on an appropriate activity. Along these lines, the ill-disposed computer game might be pointed out as:

$$
\min \max \underset{w 2 \mathrm{wa}}{\mathrm{ra}}(\mathrm{w}, \mathrm{a}) \underset{\text { a } 2 \mathrm{~A}}{\text { (Equation 9) }}
$$

Our team delivers an overall portrayal of antagonistic video games in Protocol 2. The machine learning formula selects a recipe including selection tree group, Nave Bayes, assistance angle machine, and so on. It also understands the standards of the chosen version based upon its previous idea about the enemy, as well as the recently monitored documents. Alternatively, the foe additionally goes with an action arising from its very own tenable assortment of tasks; this task is chosen. The function $\mathrm{ra}(\mathrm{w} ; \mathrm{a})$ is the motivation of the challenger. In a zero-sum The last column is the decrease percentage of the prediction error for best computer game, the benefit attribute for the pupil is $r_{l}(w ; a)=r_{a}(w ; a)$; consequently, $r_{a}(w ; a)$ is, in fact, the loss component from the student's point of

\begin{tabular}{|l|l|l|l||l||l|}
\hline Dataset & SVM & $\begin{array}{l}\text { SVM- } \\
\text { MC }\end{array}$ & -Reg & $\begin{array}{l}\text { SVM- } \\
\text { Marg }\end{array}$ & Err.Dec.(\%) \\
\hline AthR & 7.16 & 8.98 & 6.74 & 6.88 & $\begin{array}{l}5.87 \\
\text { (SVM- } \\
\text { Marg:3.91) }\end{array}$ \\
\hline & & & & & 45.49 \\
\hline BpCrypt & 2.22 & 1.52 & 2.22 & 1.21 & 18.27 \\
\hline Polar2 & 19.70 & 18.90 & 18.70 & 16.10 & 14.20 \\
\hline Subj & 12.96 & 11.76 & 13.00 & 11.12 & 19.83 \\
\hline XGraph & 9.33 & 8.51 & 9.02 & 7.48 & 23.46 \\
\hline Books & 17.43 & 16.95 & 17.35 & 13.34 & 13.74 \\
\hline Kitchen & 12.31 & 11.61 & 11.91 & 10.61 & 12.08 \\
\hline DVD & 17.55 & 16.84 & 17.61 & 15.43 & 15.06 \\
\hline Elect. & 14.01 & 13.82 & 13.91 & 11.88 & \\
\hline
\end{tabular}

\section{TABLE 1: Classification error (\%) of linear classifiers on text datasets.}

Our team matched up the efficiency of several straight weight learning algorithms utilizing Fourier fashion attributes for approximating the RBF bit along with standard RBF little bit on three datasets in Table 5.2. Our experts

monitor that the uncomplicated least-squares ( $\mathrm{LS}+\mathrm{F}$ courier) technique outpaces the particular RBF bit on two datasets just on its own. Having said that, when it is mixed together with the marginalized SVM, it exceeds all various other techniques. We similarly take note that -regularization always exceeds the regimen little bit SVM on these datasets. Possessing claimed that it does not work virtually and likewise the marginalized SVM on the Fourier basis. Our business partner this difference to the easy fact that regularization is restricted to merely making use of assistance vectors coming from the authentic dataset, unlike the marginalized SVM. The most significant increase is obtained on M27, where the training and also testing is administered on examples together with sizable missing parts. This simple truth suggests that failure might also be functional for learning with missing out on info in non-linear styles. Have straight resolved this concern for direct types utilizing a relaxation-based technique.).

TABLE 2: Classification error (\%) of approximated

\begin{tabular}{|c|c|c|c|c|c|c|}
\hline \multicolumn{2}{|c|}{ DatasetRBF.SIM } & $\begin{array}{l}\text { IS } \\
\text { (Fonirie) }\end{array}$ & \multicolumn{2}{|c|}{-Reg Lin.SIM } & $\frac{\text { Marg.STM }}{\text { Ponourit) }}$ & $\operatorname{ErI.Dec}(\%)$ \\
\hline MNST & 1.43 & 2.41 & 1.41 & 1.48 & 1.37 & 4.38 \\
\hline M27 & 6.31 & 5.97 & 6.05 & 5.66 & 4.93 & 27.99 \\
\hline Adult & 15.1 & 14.9 & 19.97 & 14,93 & 14.84 & 1.75 \\
\hline
\end{tabular}
RBF kernel.

Jittered features are understood to assist enhance the prediction accuracy of kernel SVMs on MNIST. The jittered pixels depend upon the geometrical place of non-zero pixels. Unlike stacked jittered functions, failure works just as properly with any kind of set permutation of the pixels (no matter the mathematical shape of digits), consequently dropout instruction is actually different than learning along with extra digital attributes. Both strategies may be applied concurrently, yet in this work, our team wish to assess the amount of improvement that may be attained only by applying dropout noise.

TABLE 3: Classification error(\%) arc for various measurements parts of MNIST. Reviewing no-dropout standard RBF to Monte-Carlo dimension dropout and Reg.

\begin{tabular}{|l|l|l|l|l|l|l|l|}
\hline Training size & 1000 & 2500 & 5000 & 10000 & 25000 & 50000 & 60000 \\
\hline No-D0 & 6.84 & 4.70 & 3.54 & 2.85 & 2.10 & 1.53 & 1.43 \\
\hline -Reg & 6.85 & 4.69 & 3.57 & 3.05 & 2.07 & 1.49 & 1.41 \\
\hline DO & 6.44 & 4.26 & 3.34 & 2.65 & 1.95 & 1.50 & 1.40 \\
\hline
\end{tabular}

\begin{tabular}{|l|l|l|l|l|l|l|l|}
\hline Err.Dec. $(\%)$ & $5.85 \%$ & $9.36 \%$ & $5.65 \%$ & $7.02 \%$ & $7.14 \%$ & $4.58 \%$ & $2.80 \%$ \\
\hline
\end{tabular} 
For measurement failure, our professionals can successfully marginalize the failure impact on the piece at the prophecy possibility. In Appendix C, our experts obtain the marginalized forecast performance. Table 5.3 courses result for versions of RBF SVMs on MNIST. Our team differs from the guideline dimension originating from thousand to 60,000 scenarios. Generally, failure reveals a little bit of however standard renovations over no failure with training instances. Usually, instruction, along with failure audio, leads to a $5.77 \%$ reduction in error. For considerably smaller sized quantity of examples, prediction with supposed little carries out far better than all numerous other methods. -Reg was slightly a lot more exact than no failure for much bigger training sets. For the Monte-Carlo operations, our experts utilized one hundred noisy duplications of the direct treatments. For the little bit approaches: our experts used ten loud replications training components of dimensions thousand, 2500, 5000, as well as 10000; 6 replications for instance size of 25000; and also, three duplications for the instances measurements 50000 as well as also 60000. Much bigger bunches of replications become significantly pricey, because of the improved bunch of aid angles as well as more prominent kernel sources. In experiments with three replications of 60,000 occasions, the accuracy for reared to $98.61 \%$, which advises additional duplications, can lead to much higher revelation precision.

\section{RESULTS}

While past outcomes provide that learning with failing screams can quickly build up the preciseness of neural devices and likewise found out relapse, our task verifies that dropout prepping may conveniently enhance the foresight preciseness of SVMs. Within this component, our team showed two brand new procedures that capitalize on dropout soaking up without absolutely taking examinations coming from a commotion spreading. These methods also take gently the result of a failure in the bottom as well as additionally dual preparings (- Regularization) of the SVM remodeling system. Each of these methods is straightforward in addition to effortless to execute. The evaluation results program that these methods regularly outflank standard SVMs. These outcomes are the very initial project to make the most of the failure to build up the presentation of SVMs in addition to non-straight bits. Our company introduced a pair of types of failure along with parts and additionally tentatively revealed their efficiency. Our specialists signified that randomized section estimate could be taken advantage of in addition to reduced dropout in essential to enhance both the exhibit and also efficiency of small amount equipment.

\section{CONCLUSION AND FUTURE DIRECTIONS}

This concept offers unfamiliar curved advancement estimations for learning vigorous huge side designs. Our strategies rely on considering the AI concerns as medical improving programs that can be adequately comprehended. In the entirety of our devotions, we began from an academic program of the problem and also transformed over it to a wise and curved concern, which can be comprehended by o the-rack arched improvement strategies.

\section{Rundown of commitments}

Convex ill-disposed aggregate classification Our technique vigorously performs accumulated category in the closeness foe. The meaning is a rounded quadratic course that can be adequately explained. This setup boosted the event of aggregate distinction, no matter whether there was no illdisposed part in the exam details. Our procedure dependably hammers both non-ill-disposed as well as non-social baselines. Equivalency of ill-disposed stamina as well as regularization Our procedure makes use of the opponent's shortcoming, and adjustments over their drawback to its top quality. For every foe that is suitable for adjusting the component space, our company can presume particular regularization works that immunes the AI summation to that sort of adversary. Given that the technique merely adds additional raised regularization capacities to the first improving course's target, the little arithmetic cost is included. Ultimately, the issue can be efficient in a similar ask for as the non-hearty improvement course.

Robustness of substantial edge techniques through dropout regularization Ordinary opponents need more data regarding the surprise AI platform and don't have enough estimate possessions to figure out an ideal attack. Consequently, they consider you to check out irregular assaults. They require that a part of the unnatural improvements in the info lastly tricks the AI computation. Ultimately to be vigorous against such opponents, we can easily limit the regular misery job when info is carelessly growing. Failure readying is a fabulous version of such health conditions. We identify the regularization effect of decreased failure on straight and non-direct SVMs. Our assumption is straightforward and also raised. Tentatively our experts present that our strategy is actually reliable and that it quite often hammers usual SVMs.

\section{Future titles}

The best objective is to structure an all over the world formula for energy that relates to the large a large number of the AI estimates; nonetheless, just the vulnerability of several AI computations is focused inside and out; numerous estimations keep unexplored.

\section{Improving Adversarial Artificial Intelligence}

The electrical power of a notable number of the AI estimates isn't a great peak to the base yet. As our experts recommend in Protocol 2, the scope of mixes of specific ill-disposed and also chance-based negative instances may be concentrated through and through. Some other potential bearings in illdisposed AI are actually:

\section{Scaling-up current strategies}

Scaling up ill-disposed approaches to significant datasets keeps an open issue. An appealing move is utilizing on the web calculations that are shown to be effective in different industries of AI.

\section{Learning electrical capabilities}

On the off odds that our company can easily speculate the foe's power, our company will certainly possess an increasingly realistic version of the hostile activity. Also, our company will have the choice to utilize logical option methods to manage design non-lose-lose circumstances. Unwinding non-lose-lose situations in ill-disposed settings is an essential additional issue that should often be tended to. [14]. 


\section{Dependable usage of info about the rival}

Our experts have displayed that through manipulating opponent's restrictions, our company can efficiently structure more and more passionate calculations; yet, there is, however, various knowledge regarding how to translate the crude information regarding the opponent into essential criteria in the learning calculation. These traits put on each organized and non-organized turnout projection.

\section{Development of Existing Work to Architectural} Environments

There exist several techniques in ill-disposed AI that are aimed at certain problems. Through appropriate reflection, these methods can be summed to the more considerable training class of arranged return projection. Legitimate occasions of such techniques are lament minimization calculations; these approaches depend on wealthy mathematical establishments, as well as they are aimed to become strong versus hostile clamor-utilization lament minimization estimations for managed turnout forecast merely a handful of papers. A significant component of frustration minimization calculations is that they are, for the most part, based on some versatile online estimate, which is an excellent option for sizing up existing coordinated assumption estimations. However, lament minimization estimations can similarly gain from the work that is performed in the field of ill-disposed AI. The present lament reduction computations approve that the adversary is entirely arbitrary1. A potential improvement to lament reduction estimations can be picked up through confining the foe in a progressively realistic and feasible fashion. [15] In this proposition, our experts figured out a describing for energy through dropout regularization in customary SVMs. This technique could be encompassed to be applied to managed projection concerns additionally. This advancement needs to have even more investigation and is certainly not small due to the hardness of the improvement problems of organized learning. All the same, our encouraging result on the typical SVMs advises that lessened failure should certainly boost managed assumption likewise. Although there are some simple variations of limited enemies, which are generally from the support learning network, the prospective limitations of the enemy are indeed not focused as carefully as it's performed in illdisposed AI.

\section{REFERENCES}

1. Toutanova, K., Haghighi, A., and Manning, C. D. (2005). Joint learning improves semantic role labeling. In Proceedings of the 43rd Annual Meeting on Association for Computational Linguistics, pages 589 5596. Association for Computational Linguistics.

2. Tsai, J., Kiekintveld, C., Ordonez, F., Tambe, M., and Rathi, S. (2009). Iris-a tool for strategic security allocation in transportation networks.

3. Tsochantaridis, I., Hofmann, T., Joachims, T., and Altun, Y. (2004). Support vector machine learning for interdependent and structured output spaces. In Proceedings of the twenty- rst international conference on Machine learning, page 104. ACM.

4. Tsochantaridis, I., Joachims, T., Hofmann, T., and Altun, Y. (2006). Large margin methods for structured and interdependent output variables. Journal of Machine Learning Research, 6(2):1453.

5. Wager, S., Fithian, W., Wang, S., and Liang, P. S. (2014). Altitude training: Strong bounds for single-layer dropout. In Advances in Neural Information Processing Systems, pages $100\{108$.

6. Wager, S., Wang, S., and Liang, P. (2013). Dropout training as adaptive regularization. In Advances in Neural Information Processing Systems, pages 351\{359.

Published By:

Blue Eyes Intelligence Engineering \&

7. Wang, S. and Manning, C. (2013). Fast dropout training. In Proceedings of the 30th International Conference on Machine Learning (ICML-13), pages $118\{126$.

8. Wang, S., Wang, M., Wager, S., Liang, P., and Manning, C. D. (2013). Feature noising for log-linear structured prediction. In EMNLP, pages 1170 \{1179.

9. Wang, S. I. and Manning, C. D. (2012). Baselines and bigrams: Simple, good sentiment and topic classification. In Proceedings of the ACL, pages $90\{94$.

10. Xu, H., Caramanis, C., and Mannor, S. (2009). Robustness and regularization of support vector machines. The Journal of Machine Learning Research, 10:1485\{1510.

11. Xu, H., Caramanis, C., and Mannor, S. (2010). Robust regression and lasso. IEEE Transactions on Information Theory, 56(7):3561\{3574.

12. Xu, H. and Mannor, S. (2012). Robustness and generalization. Machine learning, 86(3):391\{423.

13. Yin, Z., Jain, M., Tambe, M., and Ordo nez, F. (2011). Risk-averse strategies for security games with execution and observational uncertainty. In Proceedings of the AAAI Conference on Artificial Intelligence (AAAI).

14. Yin, Z., Jiang, A. X., Johnson, M. P., Kiekintveld, C., Leyton-Brown, K., Sandholm, T., Tambe, M., and Sullivan, J. P. (2012). Trusts: Scheduling randomized patrols for fare inspection in transit systems. In IAAI.

15. Yu, C.-N. J. and Joachims, T. (2009). Learning structural svms with latent variables. In Proceedings of the 26th Annual International Conference on Machine Learning, pages 1169\{1176. ACM. 